\title{
Coursewares exploitation of process simulation and optimization by using $\mathrm{C}++$
}

\author{
${ }^{\mathrm{a}}$ Youjun Tao, ${ }^{\mathrm{b}}$ Lili Wang, ${ }^{\mathrm{c}}$ Qian liu, ${ }^{\mathrm{d}}$ Zhengyong Li \\ School of chemical engineering and technology China University of mining and technology Xuzhou, 221116, \\ Jiangsu, China
}

\begin{abstract}
The position of mineral processing and the importance of personnel training are described, the characteristics and implications of simulation and course optimization are also illustrated, and the advantage of using $\mathrm{C}++$ to develop courseware of process simulation and optimization is introduced, at the same time, core ideology and essence of Using $\mathrm{C}++$ model to develop mold piece are expounded. And further the application of software package is illustrated, furthermore the direction of the course construction is pointed out.
\end{abstract}

Index Terms: C++; mineral processing; process simulation and optimization; courseware; developing

(C) 2012 Published by MECS Publisher. Selection and/or peer review under responsibility of the International Conference on E-Business System and Education Technology

\section{Introduction}

Mineral processing is national leading academic, and course of process simulation and optimization is one of the leading Subjects of it, this subject is from the reformation and expanding of the course of mathematical model in coal preparation, at present, many colleges of engineering which have mineral processing all open this course. The main idea is of a high level of course using mathematical method to study principle of mineral processing procedures and equipment for separation process, it is the integration of mineral processing theory, mathematical statistics, optimization and computer science [1]. It's a very important role for process simulation and optimization of teaching and research to promote the development of mineral processing engineering, searching for the optimization conditions of industrial production process, production processes and devices amplify, and optimal control of the production process[2 3]. Through this course, you can post the data analysis, scenario comparison for graduation, that is, determine the best plan for foundation; and it delivery theoretical basis to lay the Foundation for the future in the concentrator production management, forecasting of dressing production, optimization and automation, and plays a significant role to improve the students' computer application level. Aims of the teaching of the course is that enable students to use mathematical models to solve practical problems in the dressing process, and then make use of mathematical models to simulate and optimize production process by computer simulation in the of mineral processing. Mineral processing as a national key disciplines, undertake high-level engineering design and management talents cultivation, therefore, It is very

Corresponding author:

E-mail address: ${ }^{\mathrm{a} t y j} 9000 @ 126 . c o m,{ }^{\mathrm{b}}$ Wanglili211@163.com, 
important for the teaching of professional training in the mineral processing system. To further strengthen the teaching effects and improve the quality of classroom teaching, we have developed the teaching package and CAI courseware[4 5].

Through the use of combination teaching packages and CAI courseware, it can set teaching experiment on the computer, presentation, and use in one model, can make analysis for a lot of theoretical knowledge concepts interpretation of mathematical methods and the actual process in the classroom teaching process, but also verify the model instance, and make multimedia teaching come true[6].

\section{Overall design}

The teaching software includes regression analysis, optimization, interpolation, database adjustment, calculation and optimization of gravity separation, flotation simulation and optimization, crushing and sieving simulation and systems maintenance. Where regression analysis, optimization and interpolation mainly involve some typical numerical computing and language implementation of simulation methods, and gravity separation calculation and optimization, flotation simulation and optimization and crushing and sieving simulation are primarily concerned with practical application of mathematical models in mineral processing. Specific modules include:

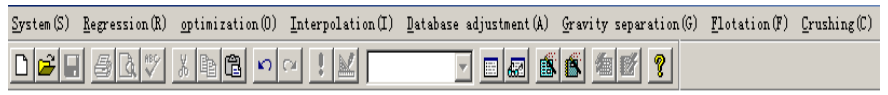

Figure 1. Specific modules

System: Modeling data entry, run the program, open the data file, save data, save capture file as, exit.

Regression analysis: Linear regression, regression linearization, polynomial regression, stepwise unit drop regression.

Searching calculate: Single variable optimization, multivariable optimization.

Interpolation: Lagrange's interpolation, cubic-spline interpolation, cubic-hermite interpolation.

Database adjustment: Gerber test calculation for triadic product.

Gravity separation calculation: Calculation of gravity separation work with two products, calculation products by dichotomy, Washability curves data, normal integral curve, distribution curve fitting.

Optimization calculation of gravity separation dense media separation optimization with classification, optimization of the main and re-election jig, actual Washability curves calculation.

Flotation Simulation: Calculation of product flotation unit, establishment of empirical flotation model.

Crushing model: Building matrix model.

System help.

Take the menu of calculation to gravity preparation for example:

\begin{tabular}{|l|}
\hline Gravity separation $(G)$ \\
\hline Calculation for two products \\
Calculation products by dichotomy \\
Washability curves calculation \\
Partition curve fitting \\
\hline
\end{tabular}

Figure 2. Menu of gravity preparation calculation

Just choose one of the terms above, and then you can get a model. 


\section{Establishment of class and using of model}

The core module of teaching package uses combined language. Because combined language have a flexible, efficient, portable and many other good advantage, and has become the most commonly usable programming languages in the development of computer software. The interface of software package system is developed by using the VC / MFC, which use a simple mode of operation. And its straight forward interface brings the teaching, model validation and function of model use together. The software has a great advantage of the objectoriented programming ideas of $\mathrm{C}++$ us, and well organizes the link between class and class. Template programming is the feature to software, and from large object template to small function template, all sufficiently make use of the advantage of the template, and which provides a sufficient condition to the software extension. At the same time, paves the way for programming specifications in the future.

Though Software development is a big workload, it has clear structure and good scalability.

In the process of program development, first, we should do systematic and comprehensive consideration and design, then conduct class design and build based on object-oriented design method of programming, in the meantime, use $\mathrm{C}++$ virtual functions and polymorphism to implement the package features. Design of class mainly includes the following:

Creat class CitemBase: public Cobject, to encapsulate all the models having properties and methods.

Creat class CItem1: public CItemBase......class CItem21 : public CitemBase, to declare and define private properties and methods to each model.

Creat class CmodalDataDlg: public Cdialog, aim to receive modeling data.

Creat class CuseModalDlg: public Cdialog, aim to receive data when we use the model.

Creat class CdisplayWnd: public CWnd, to display the modelling results.

Creat class CmyDoc: public Cdocument, to manage the models action.

Creat class CmathMethodView: public ChtmlView, to display mathmatic method of the models.

Creat class CsourceCodeView: public ChtmlView, to display robocode of the models.

At the same time,each model adopts a common template, to rewrite in accordance with their own conditions, the model as follwed :

CItemXX::CItemXX()

$\{$ InitData(); $\}$

The above function is called when the object is instantiated, the aim is to initialize the data of each model.

Model results are displayed as follows template:

CDisplayWnd::CDisplayWnd() //creat function and make current experimental model empty, whether use model or not is right

$\left\{m \_\right.$ItemBase $=N U L L$; m_bIsDisplayResult=TRUE; $\}$

int CDisplayWnd::OnCreate(LPCREATESTRUCT IpCreateStruct)

$\{\quad$ if (CWnd::OnCreate(lpCreateStruct) $==-1$ ) return -1 ;

CRect rect,btnrect; $\quad$ //creat button on the left food of the window

GetClientRect(\&rect); $\quad / /$ rect get rect range of current window

btnrect.top=rect.bottom-40;// Set the rect range of button,locate in the locatelower right of clientarea

btnrect.bottom = rect.bottom-10;

btnrect.left=rect.right-100;

btnrect.right $=$ rect.right-10;

m_btn.Create(WS_CHILD|WS_VISIBLE $\mid$ WS_BORDER,

btnrect,this,IDC_BTN); //create button

$m \_b t n . E n a b l e W i n d o w\left(m \_b B t n I s U s e\right)$;

if(!m_bBtnIsUse)

m_btn.ShowWindow(SW_HIDE);// use and display button or not

SetScrollRange(SB_VERT,0,iH-400+40);// scrollBar

SetScrollPos(SB_VERT,0); 
SetScrollRange(SB_HORZ,0,iW-500+100);

SetScrollPos(SB_HORZ,0);

return $0 ;\}$

Display window should have the following characteristics:

1. At the top when shows Model results;

2. Entered "use model data" is a non-top window, and cannot be closed;

3 . Window which shows the results after use is top window;

4. Window size can't be adjustable.

Establish view class to display the content of teaching:

CSourceCodeView::CSourceCodeView()

$\{$ TCHAR IpTemp[MAX_PATH];

GetModuleFileName(NULL,IpTemp,MAX_PATH);

CString str $=$ lpTemp;

m_strCurPath=str.Left(str.ReverseFind('II'));

m_strCurPath+="||html||sourceinit.htm";

m_InitOneTime=TRUE; $\}$

The function above is to initialize the view class.

void CSourceCodeView::OnInitialUpdate()

$\left\{\right.$ if $\left(m \_\right.$InitOneTime)

\{ Navigate2(_T(m_strCurPath),NULL,NULL);

\}

m_InitOneTime $=$ FALSE; $\}$

One advantage of this software is scalable. This feature should be attributed to the overall design and detailed design in advance. The models are derived from CitemBase, all the model should have their owe features in the CItemBase declare.

\section{Method of application}

Specific modules of the software include:

\begin{tabular}{|c|c|}
\hline Systen(S) Regression (B) optinization (0) Interpolation (I) & 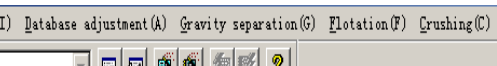 \\
\hline 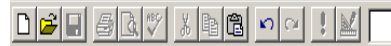 & 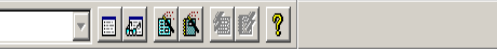 \\
\hline
\end{tabular}

Figure 3. Specific modules of the software

Take Golden-Section Search for example to explain the using method.

There are some items in system as followed:

\begin{tabular}{|l|}
\hline Enter modeling data \\
\hline Run the program \\
\hline Open the data \\
Save data \\
Data saved as \\
\hline Exit \\
\hline
\end{tabular}

Figure 4. Items in system

- Enter modeling data

When you choose a model, you can use this feature to enter the modeling data. And then it will appear a dialog box after clicking. 
First you should adjust the number of data, enter the sample data into model data column, put the other data to the corresponding data box of out-of-band data. Then click the "establish model” button, it gives the model results, and according to the model, the model data of input dialog box, the number setting, out-of-band data is different.

If the band data you enter is not correct, it will give the following tips:

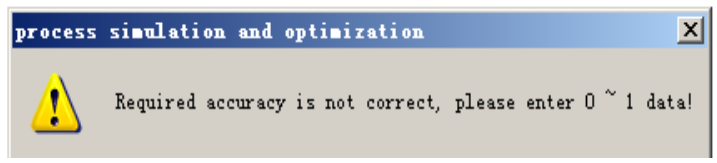

Figure 5. Dialog box for warming

- Run the program

This feature uses the system default set data model. When you select a model, directly use this feature, you can get the model results without enter data.

Figure 6. Dialog box for model results

When building popup the windows by dialog box of entering data to the function model. The raw data and calculated results will be different.

- Open the data

Open the previously stored model data. The file is *. mod. If the open file is not correct, it will appear the following tips:

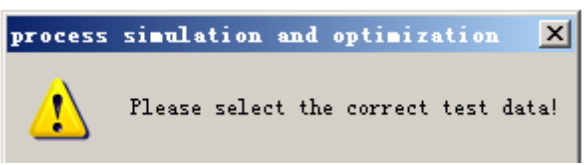

Figure 7. Dialog box for warming

- Save data

Save the current model data, file is “*.mod”.

- Data saved as

Save the current data to another file, the file is “*.mod”.

- Exit

Exit the software.

\section{Application of Teaching Software}

Simulation and optimization of course construction and teaching reform should focus on the direction of teaching content. In order to comprehensively improve the quality of classroom teaching, we should ongoing 
teaching system innovation and teaching methods, reform of teaching methods. The theory of teaching and the experiment on the computer are combined to teaching course. And its development and application for the teaching package makes the abstract mathematical theory and the simulation process of the process simulation and optimization program more intuitive and vivid. Teaching, demonstration, experiment on the computer are into this new teaching package, which is perfect in shape and function, adult in language. And From 2000 to 2002, the process simulation and optimization of the teaching and the teaching experiment on the computer in mineral processing have adopted it, which plays a well effect. By construction of the course system, we make classroom teaching and experimental teaching methods of this course more extensive, teaching contents and specific examples tight more closely. Such as, when talking about using golden section method to optimize flotation kinetic constant, we can combine the theoretical method and demonstrate of model application together well. Students can understand the process of establishing a model and the actual operating results further more clearly on the basis of abstract theoretical thinking. It also can receive the same result in explaining the applications of Lagrange's interpolation and model calculate of the election in gravity, simulation and optimization of flotation or other chapters.

\section{Conclusion}

Combined Language is the most mature and widely used language. Its flexibility and class inheritance provide a convenience to the function extension of developped software. The main purpose of simulation and optimization of teaching software development is to service classroom teaching and experiment on the computer. By means of the package and the CAI courseware, we can greatly enhance the courses level, improving teaching quality, and making the process simulation and optimization of complex theory and specific practical application complement each other.

\section{Acknowledgements}

The authors wish to acknowledge the National Natural Science Foundation of China (Grant No. 50921002), Ministry of education ("The construction project of professional characteristics ", Grant No. [2007] 25) and Ministry of finance ("national teaching team" construction project, Grant No. 2008] [19).

\section{References}

[1] Qingxiang Xu, Duanyang Zhao, and Wuheng Zuo, "Development and Application of Multimedia CAI Courseware in Engineering Mechanics Course,” Education Technology and Computer Science (ETCS), 2010 Second International Workshop on, Wuhan, pp.500-503, 6-7 March 2010.

[2] Chuyi Wei, Zhiyi Fang, Yunchun Zhang, Yunlong Jin, “A Personalized CAI Courseware System,” Computational Intelligence and Software Engineering, 2009. CiSE 2009. International Conference on, Wuhan, pp. 1-4, 11-13 Dec. 2009

[3] Chuyi WEI, Zhiyi FANG, Yunchun ZHANG and Yunlong JIN, “A Personalized CAI Courseware System” Computational Intelligence and Software Engineering, 2009. CiSE 2009. International Conference on, in Chinese, pp.1-4, 11-13 Dec. 2009

[4] A. Marascu and F. Masseglia, "Mining Sequential Patterns from Data Streams: A Centroid Approach," Journal of Intelligent Information Systems, vol. 27(3), 2006, pp. 291-307.

[5] Chuyi WEI, Zhiyi FANG, Yunchun ZHANG and Yunlong JIN, “A Personalized CAI Courseware System” Computational Intelligence and Software Engineering, 2009. CiSE 2009. International Conference on, in Chinese, pp.1-4, 11-13 Dec. 2009

[6] Feng Xilan, Chen Xiaoxia, “The Research of Design and Development of Multimedia Courseware in Engineering Mechanics,” in Chinese, Popular Science \& Technology, Nanning. 2006, (08), pp107-109. 